
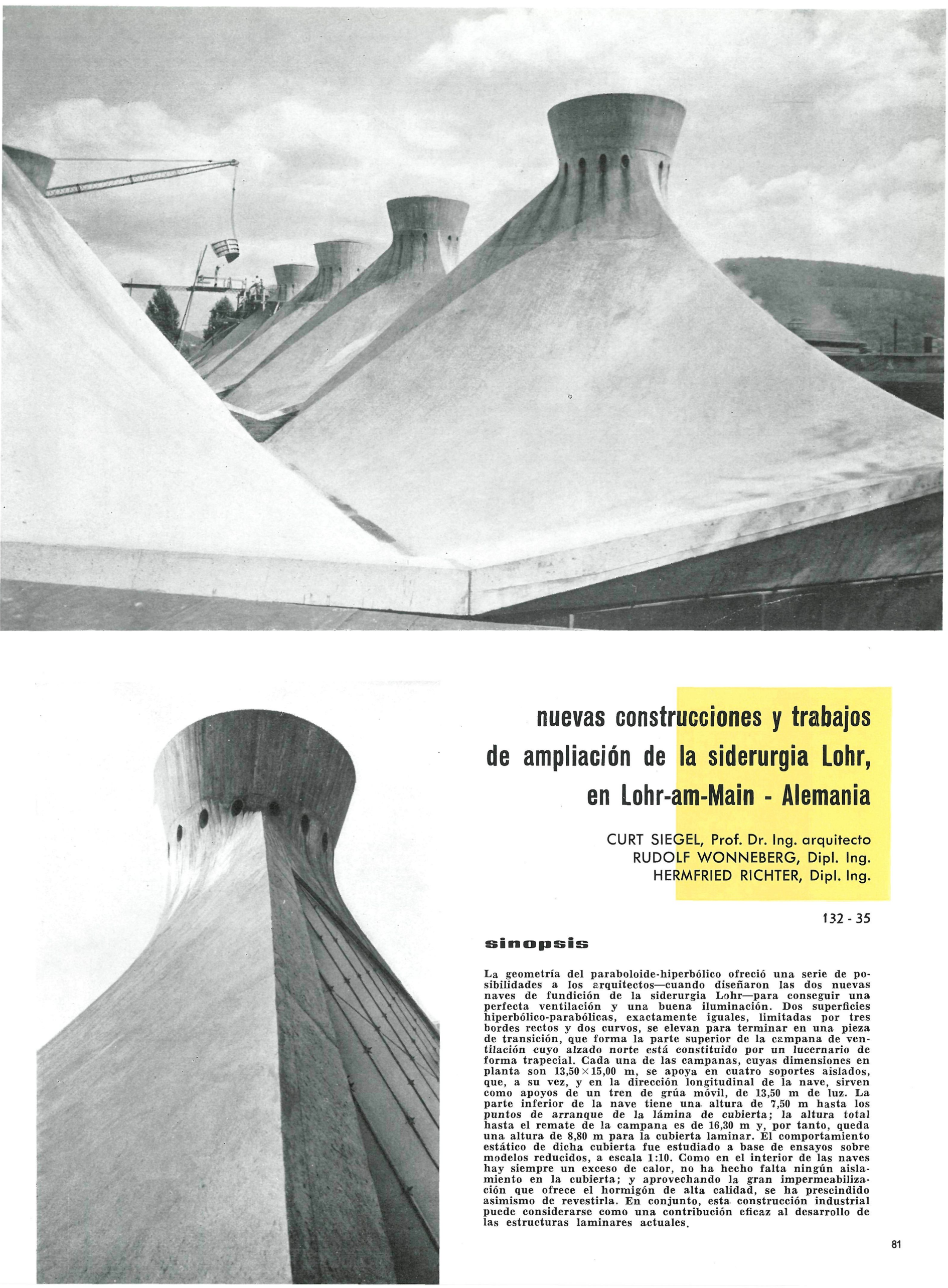

\title{
nuevas construcciones y trabajos de ampliación de la siderurgia Lohr, en Lohr-am-Main - Alemania
}

CURT SIEGEL, Prof. Dr. Ing. arquitecto RUDOLF WONNEBERG, Dipl. Ing. HERMFRIED RICHTER, Dipl. Ing.

$132-35$

\section{simopsis}

La geometría del paraboloide-hiperbólico ofreció una serie de posibilidades a los arquitectos-cuando diseñaron las dos nuevas caves de fundición de la siderurgia dohr para conseguir una piperbólico-parabólios, exa buente bordes rectos $y$ dos curvos, se elevan para terminar en una pieza de transición, que forma la parte superior de la campana de ventilación cuyo alzado norte está constituido por un lucernario de forma trapecial. Cada una de las campanas, cuyas dimensiones en planta son $13,50 \times 15,00 \mathrm{~m}$, se apoya en cuatro soportes aislados, que, a su vez, y en la dirección longitudinal de la nave, sirven como apoyos de un tren de grúa móvil, de $13,50 \mathrm{~m}$ de luz. La parte inferior de la nave tiene una altura de $7,50 \mathrm{~m}$ hasta los puntos de arranque de la lamina de cubierta; la altura total hasta el remate de la campana es de $16,30 \mathrm{~m} \mathrm{y}$, por tanto, queda una altura de $8,80 \mathrm{~m}$ para la cubierta laminar. El comportamiento estático de dicha cubierta fue estudiado a base de ensayos sobre modelos reducidos, a escala 1:10. Como en el interior de las naves hiemto un exceso de calor, no ha hecho falta ningún aislacín ou en frection aion que ofrece el hor puede considerarse como una contribución eficaz al desarrollo de las estructuras laminares actuales. 

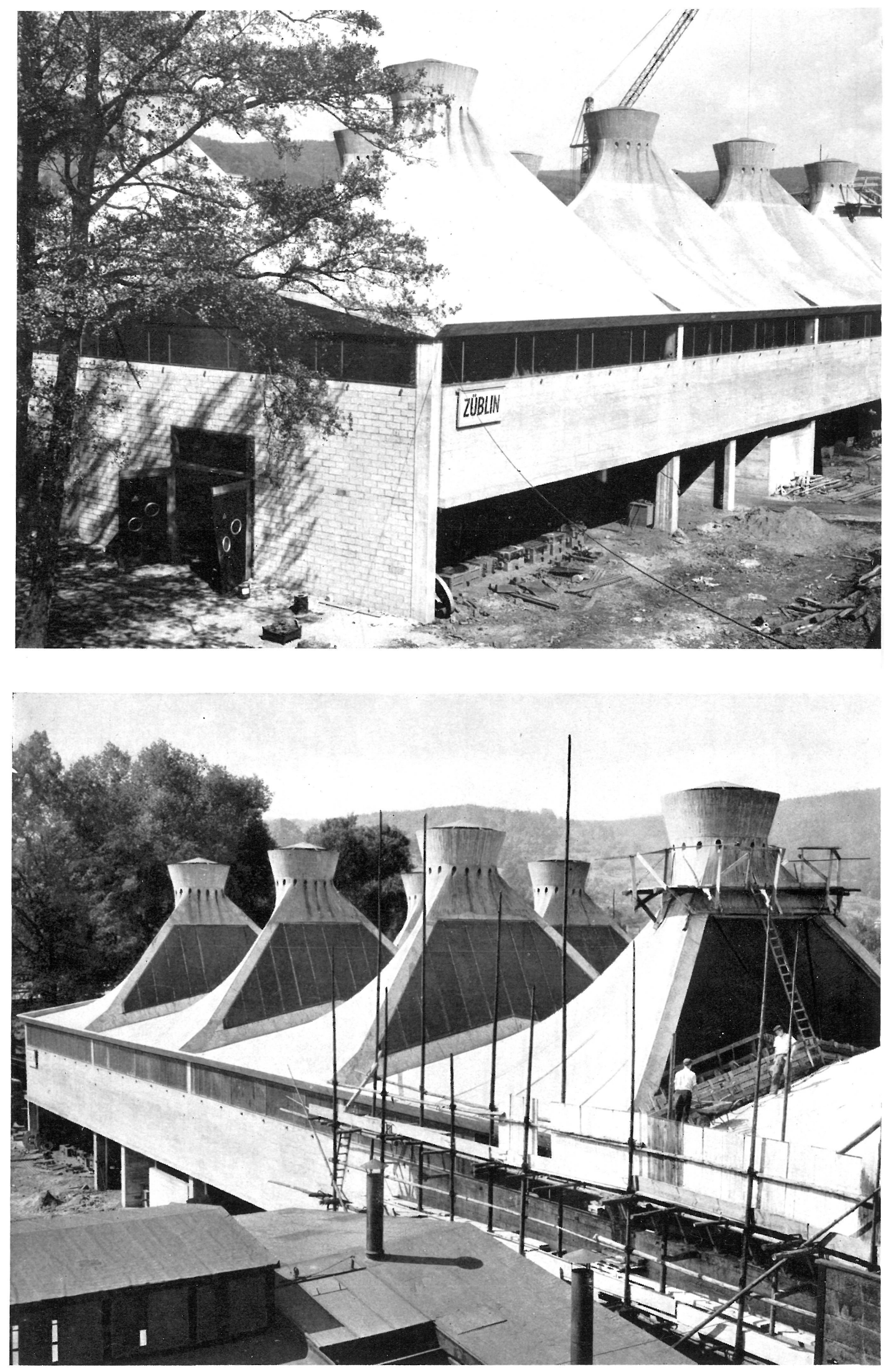
El conseguir una perfecta ventilación natural sin tener que recurrir a instalaciones especiales de maquinaria y el lograr una buena iluminación-utilizando la luz diurna, pero evitando la acción directa de los rayos solares sobre los sitios de trabajo-, fueron las condiciones básicas de las que partieron los arquitectos cuando diseñaron las dos nuevas naves de fundición para ampliación de la antigua siderurgia.

Buscaron para la cubierta una construcción cuya forma produjera el mismo efecto de succión natural que una chimenea, y que permitiera, además, colocar en el lado norte un lucernario. La geometría del paraboloide hiperbólico les ofrecía una serie de posibilidades para satisfacer dichas condiciones; por fin, al cabo de un largo estudio sobre diversos modelos, se decidieron por ella, y construyeron una cubierta laminar de hormigón armado.
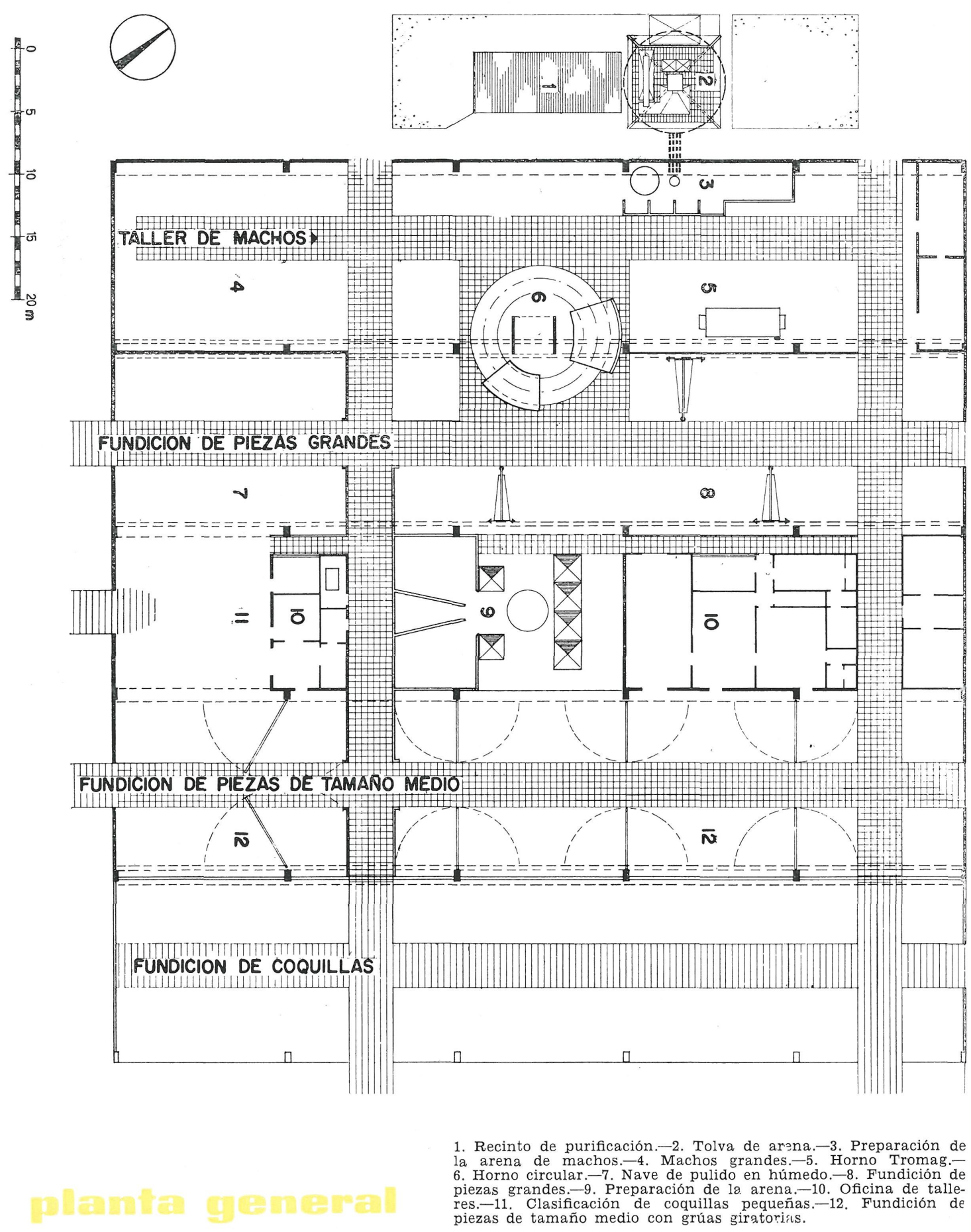

1. Recinto de purificación. - 2. Tolva de arena.-3. Preparación de la arena de machos.-4. Machos grandes.-5. Horno Tromag.6. Horno circular.-7. Nave de pulido en húmedo.-8. Fundición de res.-11. Clasificación de coquillas pequeñas.-12. Fundición de piezas de tamaño medio con grúas giratoriøs. 

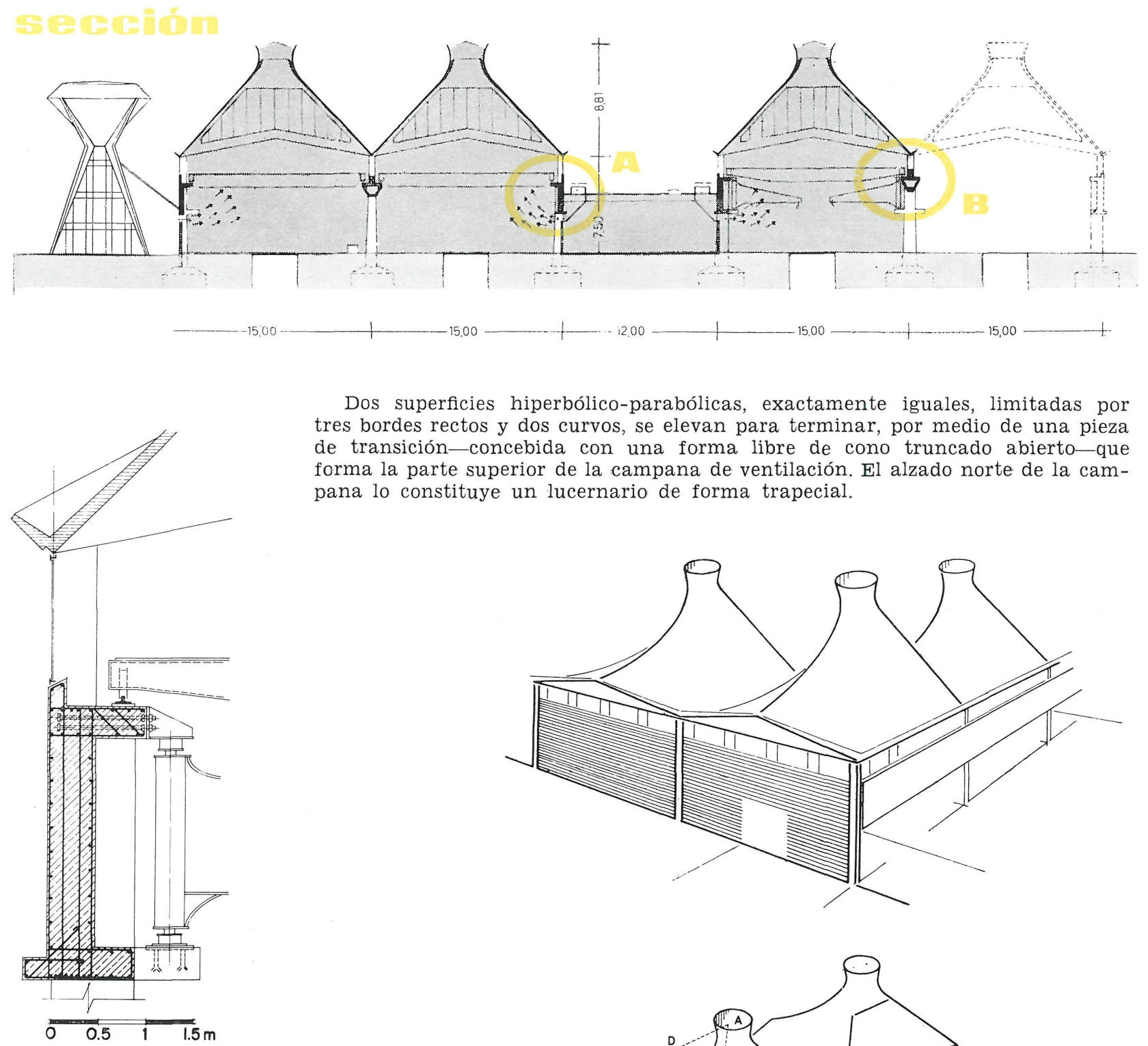

Dos superficies hiperbólico-parabólicas, exactamente iguales, limitadas por tres bordes rectos y dos curvos, se elevan para terminar, por medio de una pieza de transición-concebida con una forma libre de cono truncado abierto-que forma la parte superior de la campana de ventilación. El alzado norte de la campana lo constituye un lucernario de forma trapecial.
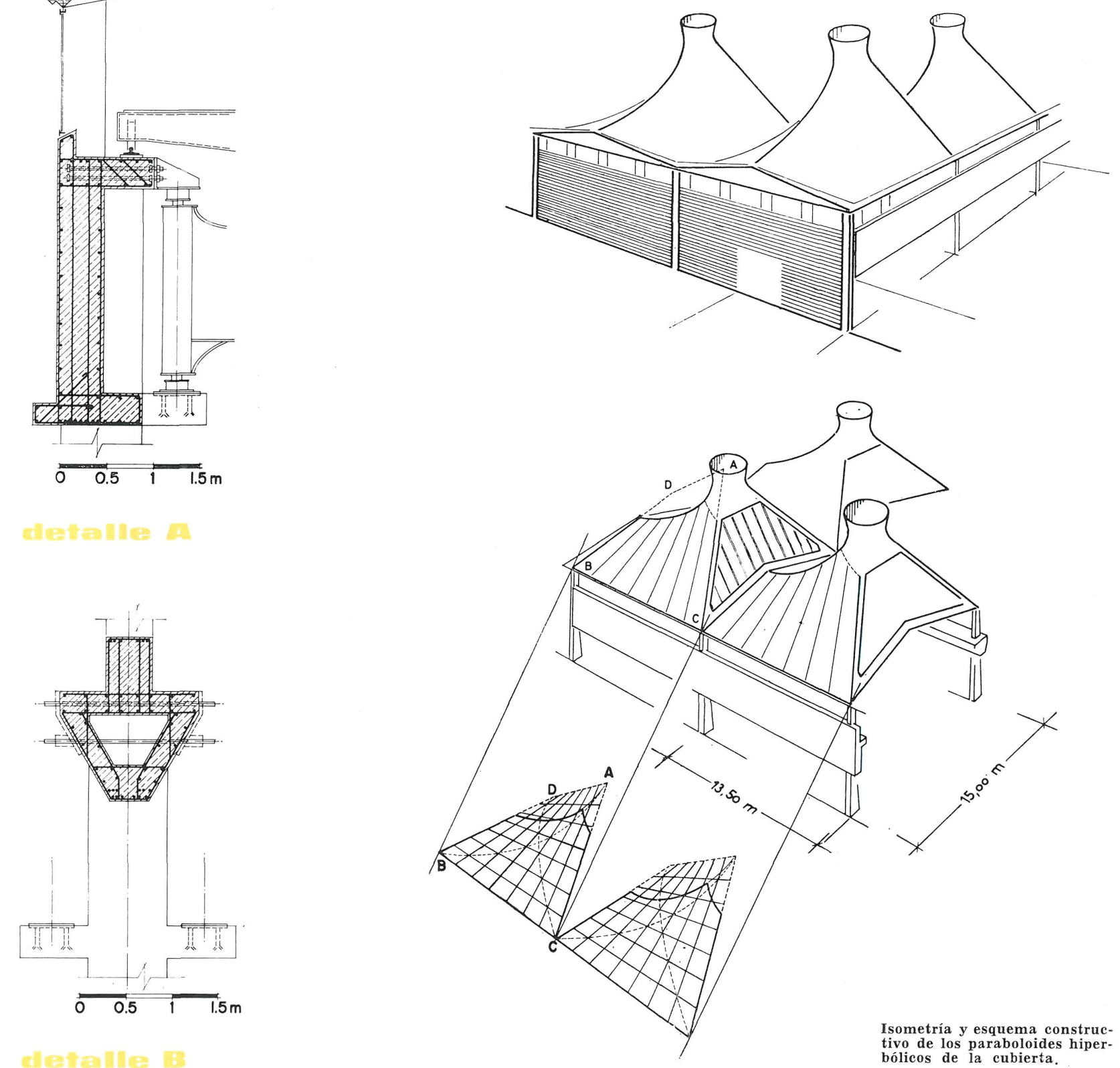


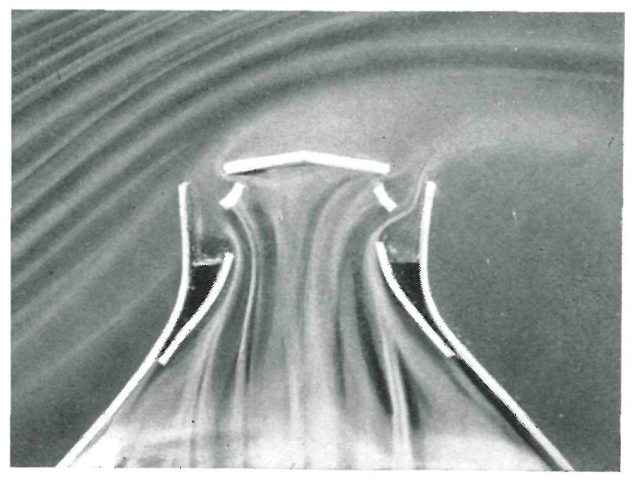

Esquema de circulación del humo en el modelo de ensayo.

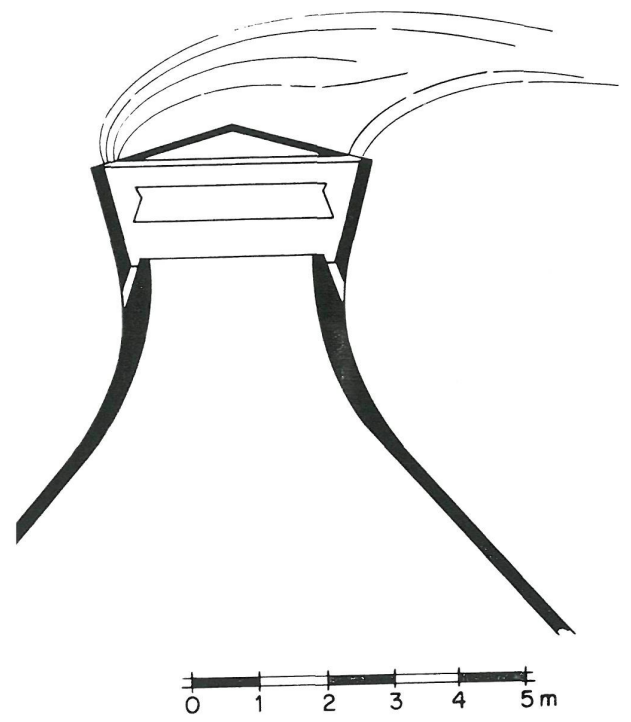

Cada una de las campanas construidas cubre una superficie de $13,50 \times 15,00 \mathrm{~m}$, y en sus puntos más bajos se apoyan en cuatro soportes aislados, que, a su vez, y en la dirección longitudinal de la nave, sirven de apoyos para un tren de grúa móvil de 13,50 $\mathrm{m}$ de luz.

La parte inferior de la nave tiene una altura de $7,50 \mathrm{~m}$, hasta los puntos de arranque de la lámina de hormigón que constituye la cubierta. La altura total de la nave, hasta el cono truncado de ventilación, es de $16,30 \mathrm{~m}$, de manera que queda una altura de $8,80 \mathrm{~m}$ para la cubierta laminar.

En la primera fase de la ampliación se construyeron cinco campanas, que ocupan una superficie total de $2.025 \mathrm{~m}^{2}$; y en la segunda, otras cinco, sobre una superficie de $15 \times 67,5$ metros.

La eficiencia del tipo de cubierta proyectada se comprobó sobre modelos, previamente a su construcción. El aire caliente, que contiene altas concentraciones de polvo y humo-producidas por el proceso de fundición-, sube sin grandes dificultades, y, deslizándose por las superficies interiores lisas de la campana, sale al exterior.

Debido al ensanchamiento en forma de embudo invertido de las campanas y a su forma externa, favorable desde el punto de vista aerodinámico, la velocidad con la que sube el aire aumenta a medida que va ganando en altura.

El aire fresco entra por las aberturas laterales, sitas en la parte inferior de la nave, y sale por la parte superior-cono truncado-, donde un artificio para la ventilación-construido

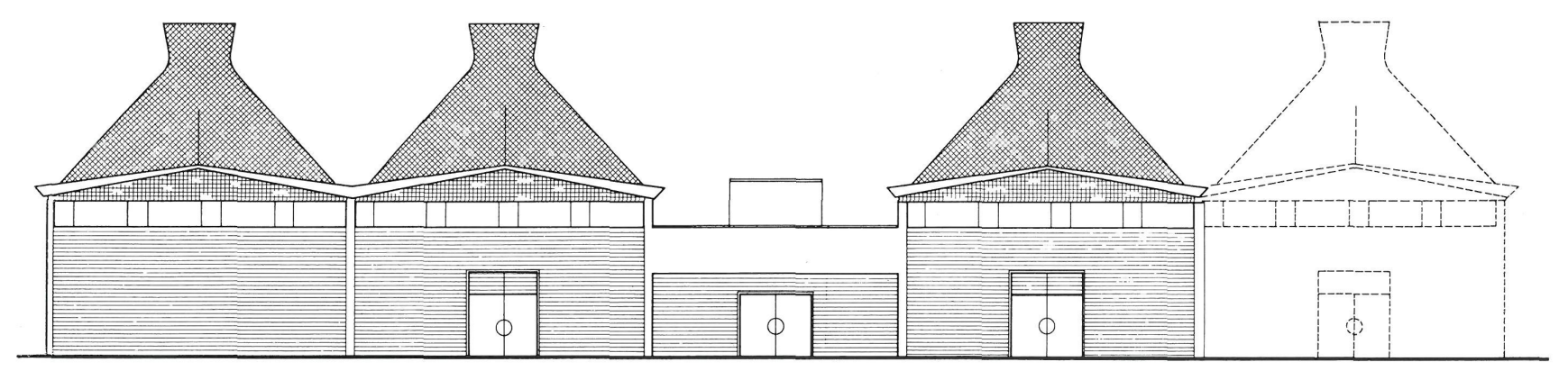

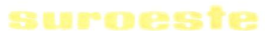

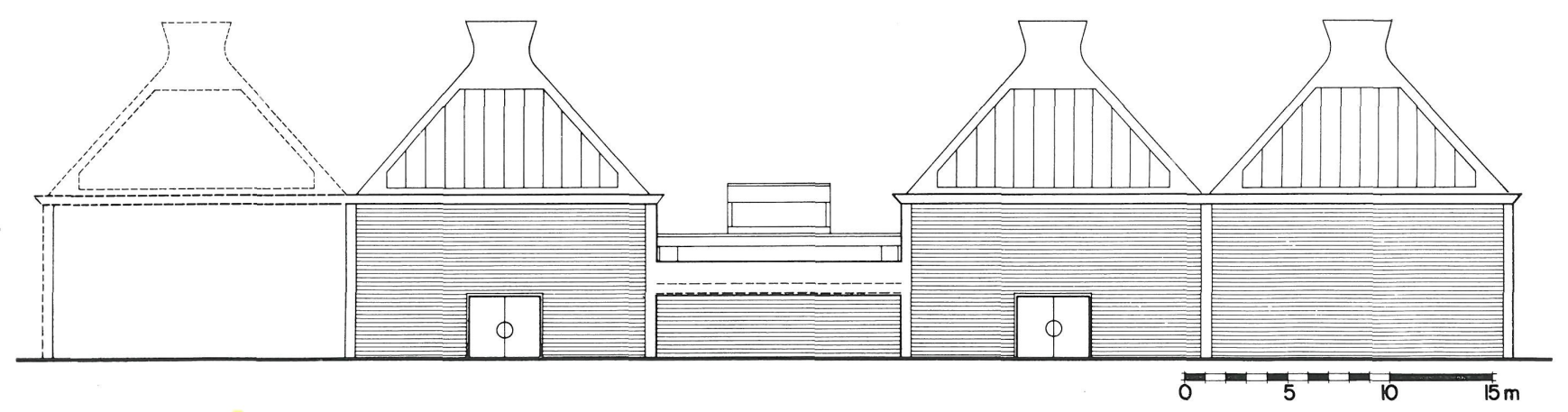



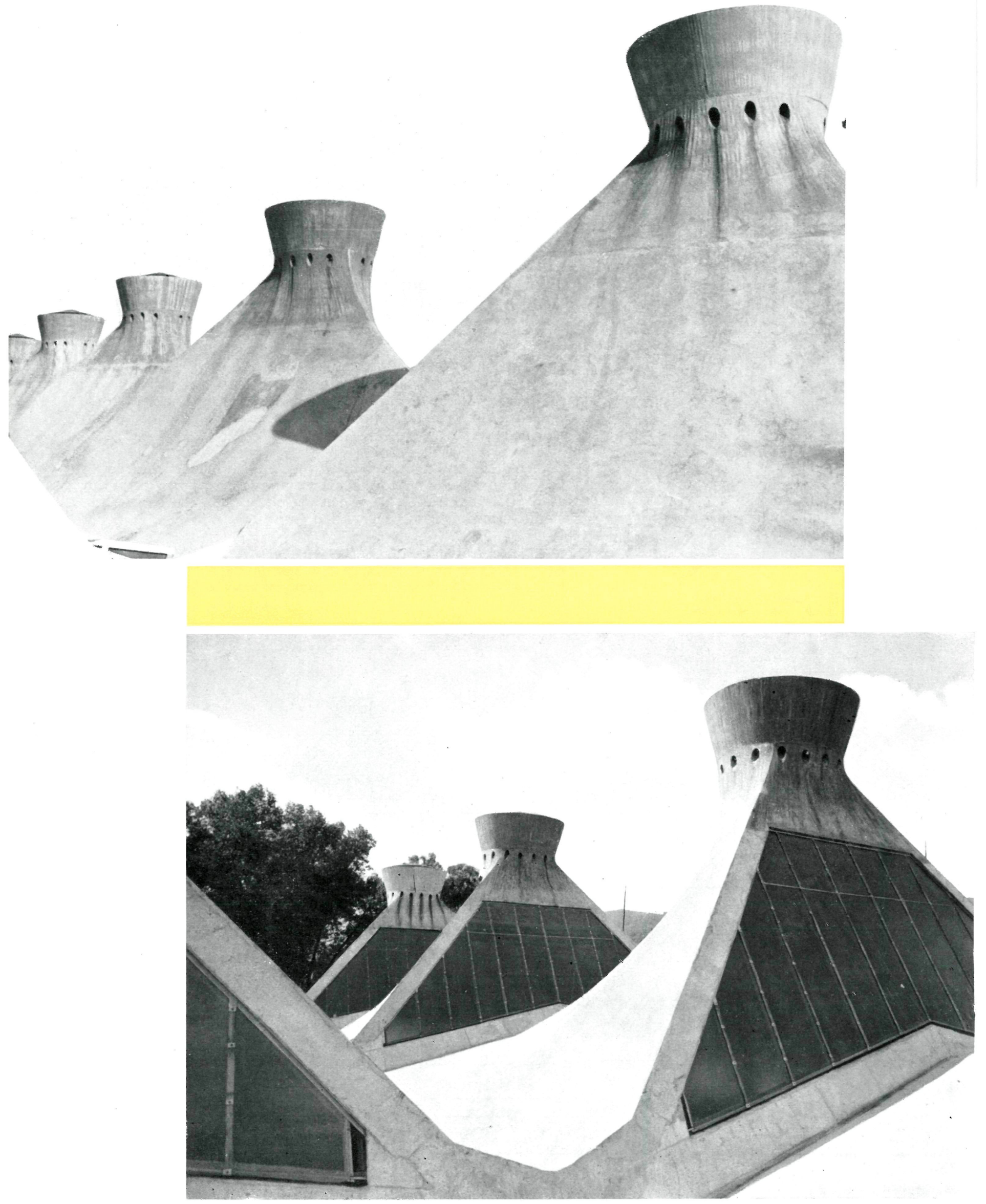


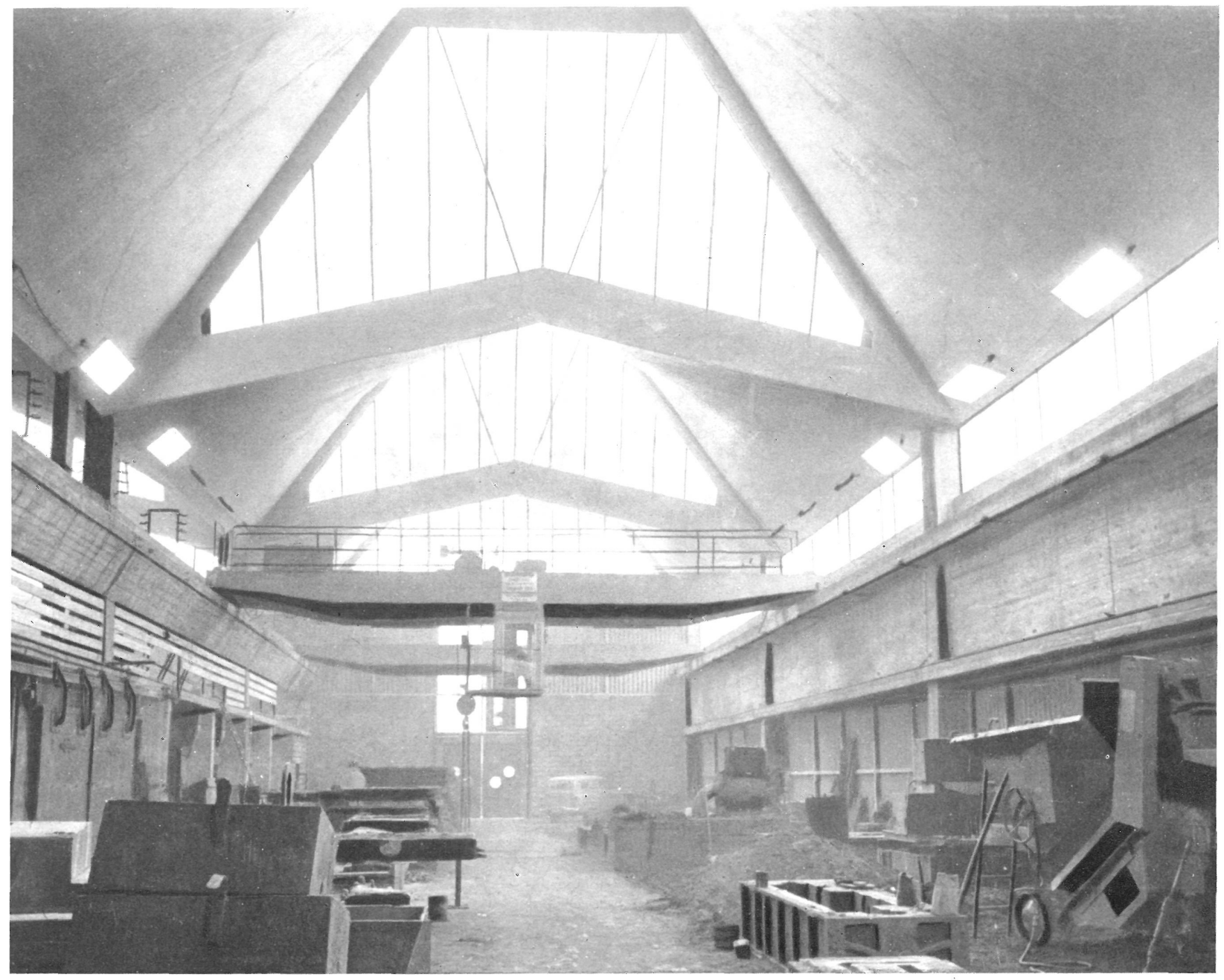

Fotos: SCHERER FRAMMERSBACH Y HIRRLINGE

a base de chapa metálica-y la forma estructural del hormigón armado en esta parte hacen que el viento y el agua de lluvia no entren en el interior de la nave.

El comportamiento estático de la cubierta fue estudiado también, en la fase de proyecto, a base de ensayos sobre modelos a escala $1: 10$. Mediante un método eléctrico para medir la dilatación se determinaron las tensiones y, consecuentemente, se dimensionaron todos los detalles constructivos.

La forma un poco difícil de la cubierta y la gran diferencia de inclinación de sus distintas partes-inclinación mínima: $20^{\circ}$, e inclinación máxima: $90^{\circ}$ en el arranque de las campanas--, se oponían a los métodos corrientes para cubrir un tejado y hacían imposible el empleo de los materiales normalmente utilizados.

Por estas razones, y aprovechando la gran impermeabilización que ofrece el hormigón de alta calidad, se ha prescindido del revestimiento de la cubierta, no sólo en sus partes más escarpadas, sino incluso en las partes más planas. Habrá ahora que esperar a comprobar si con el tiempo, al pasar los años, ese hormigón de alta calidad sigue resistiendo, sin protección alguna, al viento y a la lluvia; en caso negativo, se ha previsto la aplicación, a espátula, de una capa de resina sintética. Como en el interior de las naves hay siempre un exceso de calor, no hace falta ningún aislamiento.

El aspecto, poco frecuente, de la nueva ampliación, es consecuencia del intento de realizar una edificación atendiendo preponderantemente a la función, sin apoyarse en un ejemplo anterior o en formas preconcebidas, motivo por el cual este edificio industrial puede considerarse como una contribución importante y eficaz al desarrollo de la construcción laminar actual, y al de la arquitectura en general. 


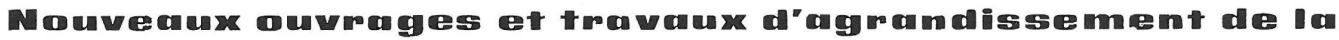

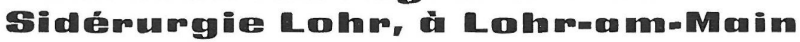

Curt Siegel, Prof. Dr. ingénieur, et Rudolf Wonneberg, Dipl. ingénæ̇eur.

La géométrie du parabolö̈de-hyperbolique a offert une série de possibilités aux architectes-lorsqu'ils dessinèrent les deux nouveaux halls de fonderie de la sidérurgie Lohr-pour assurer une parfaite ventilation et un bon éclairage.

Deux surfaces hyperbolico-paraboliques, exactement égales, limitées par trois bordures droites et deux courbes, s'élèvent pour se terminer en une pièce de transition qui forme la partie supérieure de la cloche de ventilation dont la façade nord est constituée par une lucarne trapézoídale.

Chaque cloche, dont les dimensions en plan sont 13,50 m sur 15,00, s'appuie sur quatre supports isolés qui, à leur tour et longitudinalement au hall, servent d'appuis à un train de grue mobile de $13,50 \mathrm{~m}$ de portée.

La partie inférieure du hall a $7,50 \mathrm{~m}$ de haut jusqu'aux points de naissance du voile de couverture. La hauteur totale jusq'au couronnement de la cloche est de $16,30 \mathrm{~m}$. Il reste, par conséquent, une hauteur de $8,80 \mathrm{~m}$ pour la couverture laminaire.

Le comportement statique de cette couverture a été étudié à l'aide d'essais sur modèles réduits, à l'échelle 1:10. Comme la bonne imperméabilisation qu'offre le béton de haute qualité a permis de se passer également de revêtement.

Dans l'ensemble, cette construction industrielle peut être considérée comme une contribution efficace au développement des voiles minces actuelles.

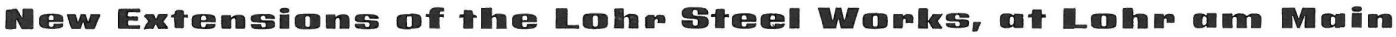

Curt Siegel, Prof. Dr. Eng., and Rudolf Wonneberg, Dipl. Eng.

The geometrical properties of the hyperbolic paraboloid provided certain advantages to the architects, when they designed the new casting mill of the Lohr steel works, especially in regard to illumination and ventilation.

Two similar hyperbolic parabolic surfaces, bounded by three straight and two curved edges, and joined together by a transition surface, form the top part of the ventilation dome. The northern elevation constitutes a skylight, of trapeze-like shape.

Each of these domes, whose plan dimensions are 13.5 by $15 \mathrm{~ms}$, rest on isolated supports, which, in turn, along the longitudinal direction of the nave, serve to support a bridge crane, with a $13.5 \mathrm{~m}$ span.

The lower part of the nave has a height of $7.50 \mathrm{~m}$, up to the springers of the roofing shell. The total height to the top of the dome is $16.3 \mathrm{~m}$, so that the shell roof has a rise of $8.8 \mathrm{~m}$.

The statical behaviour of the roof was investigated on a reduced 1:10 scale model. As there is always excess heat inside proofness of high quality concrete, in order to avoid the need of applying any external waterproofing treatment.

This project can be regarded as a significant contribution to current progress in shell roof construction.

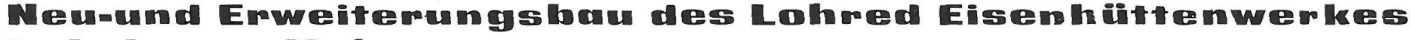 in Lolhe ane Main}

Prof. Dr. Ing. Curt Siegel und Dipl. Ing. Rudolf Wonneberg.

Die Geometrie des hyperbolischen Paraboloids gab den Architkten für den Entwurf der neuen Giessereihalle der Lohred Eisenhütte eine grosse Anzahl von Möglichkeiten zur Erzielung ausgezeichneter Lüftungs- und Beleuchtungsverhältnisse.

Zwei spiegelgleiche hyperbolisch-parabaloide Flächen mit drei geraden und zwei gekurvten Rändern setzen sich in einer Übergangsfäche fort, die den oberen Teil einer Entlüftungshaube bildet, in deren Nord-Ostseite man ein trapezförmiges
Oberlicht eingelassen hat. Jede einzelne Entlüftungshaube, deren Abmessungen $13,50 \times 15,00 \mathrm{~m}$ betragen, wird durch vier Einzelstützen getragen, die
gleichzeitig auch als Stützen für die in Hallenlängsrichtung verlaufende Kranbahn mit einer Spannweite von 13,50 m dienen.

Der Unterbau der Halle hat eine Höhe von $7,5 \mathrm{~m}$ bis zum Ansatz der Dachschale. Die Gesamthöh॰ einschliesslich der Entlüftungshaube beträgt $16,30 \mathrm{~m}$, woraus hervorgeht, dass die Dachschale selbst $8,8 \mathrm{~m}$ hoch ist.

Die Statik dieser Dachschale wurde zuvor durch Versuche an Modellen im Masstab 1:10 errechnet. Da im Innern der Hallen immer ein überschuss an Wärme herrscht, war eine Isolierung des Daches nicht notwendig. Ausserdem verzichtete man auf eine Verkleidung des Betons auf Grund seiner hohen Qualität.

Im Allgemeinen kann man diesen Industriebau als einen wirksamen Beitrag zur heutigen Entwicklung des Sehalenbaus betrachten. 